\title{
The CRFI Antagonist Verucerfont in Anxious Alcohol-Dependent Women: Translation of Neuroendocrine, But not of Anti-Craving Effects
}

\author{
Melanie L Schwandt',7, Carlos R Cortes',6,7, Laura E Kwako', David T George', Reza Momenan', Rajita Sinha', \\ Dimitri E Grigoriadis ${ }^{3}$, Emilio Merlo Pich ${ }^{4}$, Lorenzo Leggio ${ }^{5}$ and Markus Heilig*,1,6 \\ 'Laboratory of Clinical and Translational Studies, National Institute on Alcohol Abuse and Alcoholism, NIH, Bethesda, MD, USA; ${ }^{2}$ The Yale Stress \\ Center, Department of Psychiatry, Yale University School of Medicine, New Haven, CT, USA; ${ }^{3}$ Neurocrine Biosciences, San Diego, CA, USA; \\ ${ }^{4}$ Division of Brain Sciences, Department of Medicine, Imperial College London, London, UK; ${ }^{5}$ Section on Clinical Psychoneuroendocrinology and \\ Neuropsychopharmacology, National Institute on Alcohol Abuse and Alcoholism and National Institute on Drug Abuse, NIH, Bethesda, MD, USA; \\ ${ }^{6}$ Center for Social and Affective Neuroscience, Linköping University, Linköping, Sweden
}

\begin{abstract}
Blockade of corticotropin-releasing factor receptor I (CRFI) suppresses stress-induced alcohol seeking in rodents, but clinical translation remains. Here, we first showed that the CRFI antagonist verucerfont potently blocks hypothalamic-pituitary adrenal (HPA) axis activation in adrenalectomized rats. We then evaluated verucerfont for its ability to block HPA axis activation and reduce stress-induced alcohol craving in alcohol-dependent patients. Anxious, alcohol-dependent women (age 2I-65 years, $n=39$ ) were admitted to the NIH Clinical Center and completed withdrawal treatment before enrollment if needed. One-week single-blind placebo was followed by randomized doubleblind verucerfont (350 mg per day) or placebo for 3 weeks. Verucerfont effects on the HPA axis were evaluated using the dexamethasoneCRF test. Craving was evaluated using two established protocols, one that combines a social stressor with physical alcohol cue exposure, and one that uses guided imagery to present personalized stress, alcohol, or neutral stimuli. An $\mathrm{fMRI}$ session examined brain responses to negative affective stimuli and alcohol cues. In contrast to our recent observations with another CRFI antagonist, pexacerfont, verucerfont potently blocked the HPA axis response to the dexamethasone-CRF test, but left alcohol craving unaffected. Right amygdala responses to negative affective stimuli were significantly attenuated by verucerfont, but responses to alcohol-associated stimuli were increased in some brain regions, including left insula. Discontinuation rates were significantly higher in the verucerfont group. Our findings provide the first translational evidence that CRFI antagonists with slow receptor dissociation kinetics may have increased efficacy to dampen HPA axis responses. The findings do not support a clinical efficacy of CRFI blockade in stress-induced alcohol craving and relapse. Neuropsychopharmacology (2016) 4I, 28I8-2829; doi:I0.1038/npp.20 I6.6I; published online I5 June 2016
\end{abstract}

\section{INTRODUCTION}

Despite decades of research, relapse rates remain high in alcohol dependence $(\mathrm{AD}$, hereafter equated with alcoholism; Brandon et al, 2007; Hunt et al, 1971). A major objective of alcohol research is therefore to develop medications with an ability to prevent relapse to alcohol use. Preclinical studies have identified several classes of molecules with an ability to block relapse-like behaviors in experimental animals (Heilig and Egli, 2006; Litten et al, 2012). One of the conclusions that have emerged from this research is that type 1 receptors for corticotropin-releasing factor 1 (CRF1) are key mediators of stress-induced alcohol seeking. Accordingly, in rodents,

* Correspondence: Dr M Heilig, Center for Social and Affective Neuroscience, IKE Linköping University, Linköping 58I83, Sweden, Tel: +46 I3 2866 26, Fax: +46 (I0) 103 3393, E-mail: markus.heilig@liu.se ${ }^{7}$ The first two authors contributed equally to this work.

Received 4 February 2016; revised 12 April 2016; accepted 13 April 2016; accepted article preview online 25 April 2016 systemic administration of brain penetrant CRF1 antagonists consistently blocks relapse-like behavior triggered by stress (reviewed, e.g., in Heilig and Koob, 2007; Le and Shaham, 2002a; Mantsch et al, 2016; Zorrilla et al, 2013).

These and other preclinical findings with CRF1 antagonists predict an ability of these molecules to suppress stress-induced craving and relapse in humans, but clinical translation of the preclinical results has not yet been achieved. The first CRF1 antagonist to be evaluated in humans, R121919, showed promise in depression (Zobel et al, 2000), but was terminated owing to safety issues widely shared by first-generation CRF1 antagonists. CRF1 antagonists with improved safety followed, but yielded negative results both in depression (Binneman et al, 2008) and anxiety disorders (Coric et al, 2010). Recently, we evaluated the effects of pexacerfont, a potent, selective, orally available and brain penetrant CRF1 antagonist, on a battery of experimental outcomes in AD. Hypothalamicpituitary adrenal (HPA) axis responses, alcohol-craving, and fMRI brain responses to aversive stimuli in anxious patients 
with $\mathrm{AD}$ were all unaffected by pexacerfont, despite ensuring adequate central nervous system exposure (Kwako et al, 2015).

It was recently proposed that binding kinetics, and in particular the time constant of dissociation from the receptor ('off-rate'), are major determinants of CRF1 receptor antagonist efficacy (Fleck et al, 2012). Verucerfont (formerly GSK561679) is a potent, selective CRF1 receptor antagonist (IC $_{50}$ for CRF1, CRF2, and CRF-BP 6.1, >1000 and $>1000 \mathrm{nM}$, respectively), and is orally available and brain penetrant (Gilligan et al, 2009; Tellew et al, 2010). It is structurally related to compounds with slow off-rates (Fleck et al, 2012), and therefore hypothesized to possess improved efficacy over compounds with similar nominal binding affinity constants but faster off-rates, such as pexacerfont. Verucerfont lacked efficacy in a controlled trial for major depression (GlaxoSmithKline, 2010), but its effects on stressinduced alcohol craving have not been evaluated.

Here, we first compared verucerfont with pexacerfont in a rat assay that may offer a translational biomarker, blockade of ACTH release in adrenalectomized rats. We then carried out a double-blind, placebo-controlled experimental medicine study to evaluate verucerfont in treatment-seeking women with anxious AD. As primary outcome, we assessed the effects of verucerfont on stress-induced alcohol craving and on brain responses to aversive stimuli, using established procedures previously used to evaluate pexacerfont (Kwako et al, 2015). As a translational biomarker, and a measure of target engagement, we also examined whether verucerfont would block HPA axis responses in the combined dexamethasone-CRF test ('dex-CRF test'; Modell et al, 1998).

\section{MATERIALS AND METHODS}

\section{Measurement of ACTH in Adrenalectomized Rats}

Adrenalectomized rats were used as an assay to assess functional CRF1 receptor inhibition by a range of CRF1 antagonists thought to belong to two classes, with fast or slow CRF1 offset (fast: CP-316 311, pexacerfont; slow: R121919, verucerfont). In this assay, corticosterone levels become negligible following adrenalectomy, and in the absence of the negative feedback, ACTH levels increase markedly in response to CRF release. This increase is blocked by peptide CRF antagonists (Rivier et al, 1999).

Suppression of ACTH in adrenalectomized rats was assessed as previously described (Fleck et al, 2012). In brief, experiments were carried out in agreement with the NIH guidelines, and approved by the IACUC at Neurocrine Biosciences. Male Sprague-Dawley rats were received at 175$200 \mathrm{~g}$ from Charles River Laboratories (San Diego, CA) and housed in a 12-12 light cycle for 1 week before adrenalectomy. Rats were adrenalectomized at Neurocrine Biosciences, and $\mathrm{NaCl}$ was replenished. Adrenalectomy was verified by plasma corticosterone measurements (MP Biomedicals, Irvine, CA). Seven days after adrenalectomy, rats were implanted with femoral vein catheters. After $\sim 4$ days, rats were prepared for blood sampling by attaching their catheters to PE50 tubing and a syringe, and acclimated to individual opaque sampling cages for $1 \mathrm{~h}$. These cages allow sampling to occur without disturbance to the rat. Blood samples $(0.3 \mathrm{ml})$ were taken after acclimation and blood volumes were replaced with $5 \mathrm{U} / \mathrm{ml}$ heparinized saline. Blood samples were stored on ice with EDTA.
After a baseline blood sample, rats received oral doses of either vehicle at $5 \mathrm{ml} / \mathrm{kg}$ ( $5 \%$ Cremaphor EL; Sigma) or the respective drug in the same volume of vehicle. In each case, the dose was $10 \mathrm{mg} / \mathrm{kg}$, based on prior pharmacokinetic studies showing that this dose results in adequate and comparable exposure (Neurocrine Biosciences, data on file). Blood samples were taken 1, 2, 3, 4, and $6 \mathrm{~h}$ later. Plasma was separated by centrifugation at $4{ }^{\circ} \mathrm{C}$ and stored at $-80{ }^{\circ} \mathrm{C}$ for subsequent measurement of $\mathrm{ACTH}$ by radioimmunoassay (MP Biomedicals, Irvine, CA).

Data were analyzed using two-way ANOVA, with treatment as a between-subjects and time as a within-subjects factor. Individual baseline values were used as a covariate to normalize for individual and day-to-day variation in basal ACTH. This is largely equivalent to normalizing all values to baseline, but preserves the variance at $t=0$.

\section{Clinical Study: Subjects and General Procedure}

Prospective participants were recruited through advertisements and referrals between November 2010 and March 2015. Subjects were phone screened, and admitted to the NIH Clinical Center in Bethesda, MD where they underwent medically managed withdrawal if needed. Once they had an undetectable breath alcohol concentration and did not require benzodiazepines for alcohol withdrawal, they were evaluated for eligibility. Detailed eligibility criteria are available at: http://www.clinicaltrials.gov/ct2/show/NCT01187511.

In brief, subjects were females between 21 and 65 years old, diagnosed with $\mathrm{AD}$ according to the Structured Clinical Interview for DSM-IV (SCID; First et al, 1996), had scores $>39$ on the Spielberger State Trait Anxiety Inventory-Trait Version (STAI; Spielberger et al, 1970), and were in good physical health. They were excluded if they had complicated medical or psychiatric problems or were unable to participate in study procedures or to provide informed consent. Similar to previous studies with verucerfont (GlaxoSmithKline, 2010; Grillon et al, 2015), males were excluded because preclinical safety data indicate a potential for testicular toxicity with this molecule. Informed consent was obtained as approved by the NIH Institutional Review Board.

Out of 44 individuals enrolled, 39 completed the study (Figure 1a). Owing to procedural reasons (such as drop out due to adverse events, or a nation-wide pharmacy shortage of CRF during a period of time), the $n$ with available data for the respective outcome differs, and is indicated in the legend of the corresponding figure. The original target sample size of 50 completers was chosen to detect an effect size of Cohen's $d \geqslant 0.8$ with a power $\geqslant 0.80$ at a two-tailed $\alpha$ of 0.05 , similar to what was reported under laboratory conditions using the clinically approved alcoholism medication naltrexone (O'Malley et al, 2002). However, the occurrence of several adverse events, combined with negative results in a similar protocol with pexacerfont prompted a futility analysis. This indicated a very low probability of finding a significant effect on the primary craving outcomes if recruitment were continued to reach 50 total subjects, and the study was halted.

Subjects were randomized to verucerfont or placebo using a double-blind parallel group design with a 1:1 allocation. A 1-week single-blind placebo lead-in period was followed by 3 weeks of double-blind randomized treatment, where 


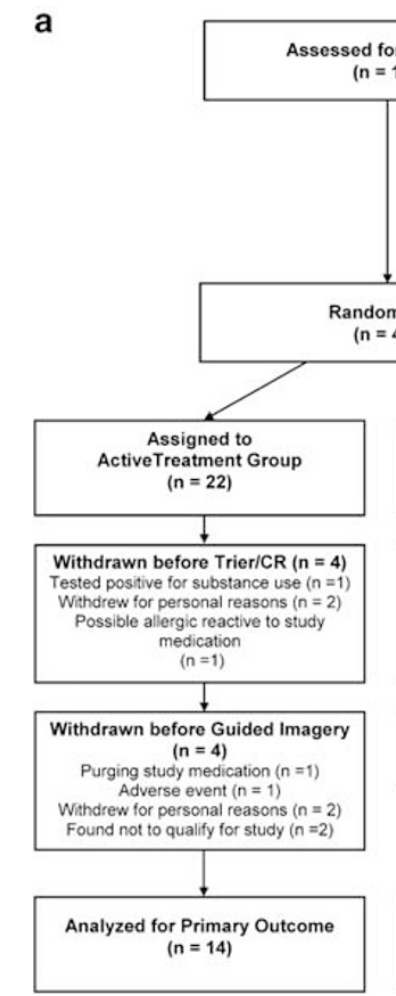

\section{for Eligibility}

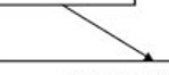

Excluded $(n=98)$ Did not meet study criteria
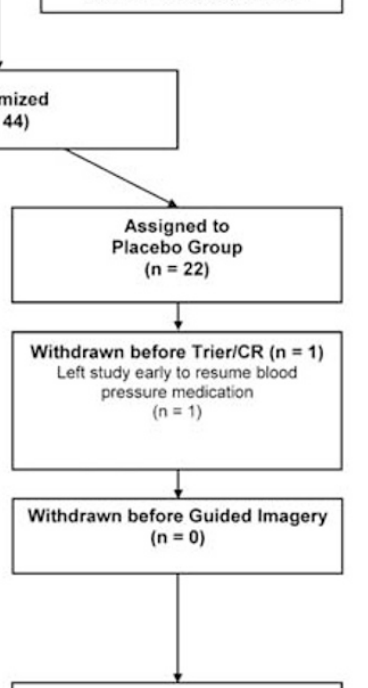

Analyzed for Primary Outcome ( $n=21$ )

Figure I (a) CONSORT graph for the clinical study. (b) Timeline for procedures and data collection during challenge sessions used to provoke alcohol craving, subjective distress, and neuroendocrine responses used as biomarkers in this experimental medicine study. Upper panel: sessions using a combination of a social stress task and presentation of physical alcohol cues (Trier Social Stress/cue-reactivity test 'Trier/CR'). Lower panel: sessions using guided imagery induced by auditory scripts.

subjects received either a fixed dose of $350 \mathrm{mg}$ per day of verucerfont or placebo. All subjects remained hospitalized throughout the study, and participated in standard-of-care behavioral $\mathrm{AD}$ treatment. Upon inclusion, they were evaluated for $\mathrm{AD}$ severity using the $\mathrm{AD}$ Scale (ADS; Skinner, 1984), for family history of AD using the Family Tree Questionnaire (FTQ; Mann et al, 1985), for addiction severity phenotypes using the Addiction Severity Index (ASI; McLellan et al, 1980), for alcohol consumption in the past 90 days using the Timeline Follow-Back (TLFB; Sobell et al, 1986), for alcohol craving using the Penn Alcohol Craving Scale (Flannery et al, 1999), for depression and anxiety symptoms using the Comprehensive Psychopathological Rating Scale (Asberg and Schalling, 1979), for personality traits using the NEO Personality Inventory Revised (NEO; Costa and McCree, 2002), for post-traumatic stress disorder (PTSD) symptom severity using the PTSD Symptom Severity Interview (Foa et al, 1993), and for early life adversity using the Childhood Trauma Questionnaire (CTQ; Bernstein et al, 1994). For the experimental procedures, smokers were allowed ad lib smoking until $1 \mathrm{~h}$ before the respective procedure, and were then required to abstain from smoking until completion of that procedure.

\section{Neuroendocrine Testing}

To assess the ability of verucerfont to block CRF-induced ACTH and cortisol release, subjects underwent a dex-CRF test on days 5 (during placebo lead-in) and 29 (during randomized treatment), carried out as described previously (Rydmark et al, 2006). ACTH and cortisol were assayed using standard CLIA-certified clinical assays by the Department of Laboratory Medicine, NIH Clinical Center. Effects of verucerfont were evaluated on day 29; baseline data were only used for exploratory analyses. In addition, we collected morning cortisol samples twice weekly.

\section{Behavioral Challenge Sessions}

Alcohol cravings and emotional responses were assessed using two established procedures: (1) personalized auditoryguided imagery scripts (hereafter referred to as scripts), and (2) the combined Trier Social Stress/cue-reactivity test (hereafter referred to as Trier/CR). The scripts procedure is described in detail elsewhere (Kwako et al, 2014; Sinha et al, 2011a); briefly, it consisted of three sessions that used personalized auditory-guided imagery scripts, each $\sim 5$ min in duration, to present stress-, alcohol cue-associated or neutral stimuli on days 24-26. The order of the script types was counterbalanced across subjects. The Trier/CR combined the Trier Social Stress Test (Kirschbaum et al, 1993) with exposure to physical alcohol cues, that is, handling and smelling, but not consuming the subject's preselected preferred alcoholic beverage (Stasiewicz et al, 1997). This combined challenge was carried out as described previously (George et al, 2008; Kwako et al, 2014), around day 21 of the study. Both challenge procedures (i.e., Trier/CR and scripts) 
began at 1500 hours to minimize differences in circulating cortisol.

During the challenge sessions, craving for alcohol was rated using the AUQ (Bohn et al, 1995). The STAI (Spielberger et al, 1970) and the Subjective Units of Distress Scale (Wolpe, 1969), a visual analog scale ranging 1-100, were used to assess anxiety and emotional responses. ACTH and cortisol were used as endocrine stress markers. A timeline for experimental manipulations and data collection in the two challenge procedures is shown in Figure 1B.

\section{Functional Imaging}

Around day 23 of the study, subjects underwent an fMRI scan. Because scanners were replaced at the NIH Clinical Center during the course of the study, subjects were scanned on three different scanners: 3T General Electric MRI scanner, 3T Siemens Skyra scanner, and a 3T Siemens Verio scanner. To control for potential variability, we included a scanner variable as a covariate in all analyses. Imaging paradigms included presentation of 130 negative, positive, and neutral pictures from the International Affective Picture System (IAPS; Lang et al, 1999). Scrambled images were used as the control condition and displayed during the interstimulus interval (ISI; $1.5-14$, average $3.2 \mathrm{~s}$ ). The scrambled images, derived from IAPS images, preserved brightness and color but did not contain recognizable features. Images were presented in random order in one run lasting $8 \mathrm{~min}$. A second paradigm presented 90 pictures of alcoholic and neutral beverages (e.g., milk, orange juice) in random order in a run lasting $6 \mathrm{~min}$. Finally, 130 emotional (fearful, angry, happy, and neutral) faces (Matsumoto and Ekman, 1988), or a non-emotional control cross-hair (ISI), were presented for $8 \mathrm{~min}$, in random order. Whole brain images were collected for $22 \mathrm{~min}$.

fMRI data were analyzed using Analysis of Functional Neural Images (AFNI) software (Cox, 1996). Statistical maps were generated for each individual by linear contrasts between regressors of interest (negative and positive IAPS images; alcoholic and non-alcoholic beverages; happy, neutral, and fearful faces). Preprocessed time-series data for each individual were then analyzed by multiple regression, which allowed covariation of variables related to head motion and other relevant factors such as subject age. We also calculated a statistical map of the activation within each group (verucerfont and placebo) for each contrast of interest. Each condition was compared with the baseline scrambled image. The individual $\beta$-coefficients calculated from the general linear model for each condition were entered into a second-level random-effect factorial analysis using the AFNI multivariate modeling program $3 \mathrm{dMVM}$ to test for differences between the verucerfont and placebo groups. Models included covariate baseline characteristics for which trends for differences between randomization groups were found: race, ADS score and CTQ. Correction for multiple comparisons across the whole brain was conducted using Monte-Carlo simulations run via 3dClustSim in AFNI_16.0.19, the current version, which computes the voxel-probability and minimum cluster-size threshold needed to obtain a 0.05 family-wise error-corrected $\alpha$-value.

\section{Statistics}

Rat endocrine data were analyzed using the GLM module in STATISTICA version 12.0 (StatSoft, Tulsa OK), and two-way ANOVA with treatment as a fixed, between-subjects factor, and time as a repeated-measures, within-subject factor. Human behavioral data were analyzed using PROC MIXED for mixed-effect modeling in SAS version 9.3 (SAS Institute, Cary, NC), with treatment (verucerfont $v s$ placebo) as the fixed, between-subjects factor. Repeated-measures, withinsubjects factors included script condition (neutral, alcohol cue, or stress) and time point (Scripts outcome measures), or just time point (Trier/CR outcome measures). Significance was set at $P<0.05$ for all tests, and all post hoc comparisons were conducted using Tukey's honestly significant difference test. Potential covariates were evaluated on a model-bymodel basis such that covariates that significantly predicted the outcome measure were retained in the model. Covariates that were evaluated included age, race, years of education, lifetime diagnosis of PTSD from the SCID, ADS score, family history density from the FTQ, total score from the ASI, number of heavy drinking days and average number of drinks per drinking day from the TLFB, total score from the CTQ, neuroticism score from the NEO, and trait anxiety at baseline from the STAI. Model-specific covariates are noted in the relevant figure legends. The Kenward-Roger correction (Kenward and Roger, 1997) was used in all models, as the use of this correction is highly recommended in repeated-measures models with more complex covariance structures, especially when there is an unbalanced design (Littell et al, 2006). We note that this correction may result in atypical denominator degrees of freedom compared with traditional repeated-measures models (e.g., denominator degrees of freedom may actually be higher than the number of subjects).

\section{RESULTS}

\section{In vivo Inhibition of ACTH in Adrenalectomized Rats}

The small-molecule, non-peptide CRF1 receptor antagonists varied markedly in their ability to suppress ACTH in the adrenalectomized rat assay, as shown by a highly significant main effect of treatment $(\mathrm{F}[4,28]=43.2, \quad P<0.001$; Figure 2A). Post hoc analysis showed that the prototypic non-peptide CRF1 receptor antagonist NBI30775 (R121919) and verucerfont were both significantly different from vehicle, CP-316311, and pexacerfont $(P<0.001$ for all comparisons collapsed across time-points); the latter three treatments in turn did not differ from each other. A differential effect of treatments over time was also shown by a significant treatment $\times$ time interaction $(\mathrm{F}[20,140]=6.4$, $P<0.001)$. Accordingly, detailed post hoc analysis showed that both NBI30775 and verucerfont inhibited ACTH release throughout the following $6 \mathrm{~h}$ of measurement $(P<0.001 v \mathrm{~s}$ vehicle at each time-point, and $v s$ the respective pretreatment baseline). In contrast, CP-316 311 and pexacerfont did not suppress ACTH levels at any time-point, and neither compared with vehicle nor with their own baseline.

Thus, for reference compounds, data were in excellent agreement with previously published results (Fleck et al, 2012). The efficacy of verucerfont in suppressing ACTH in 


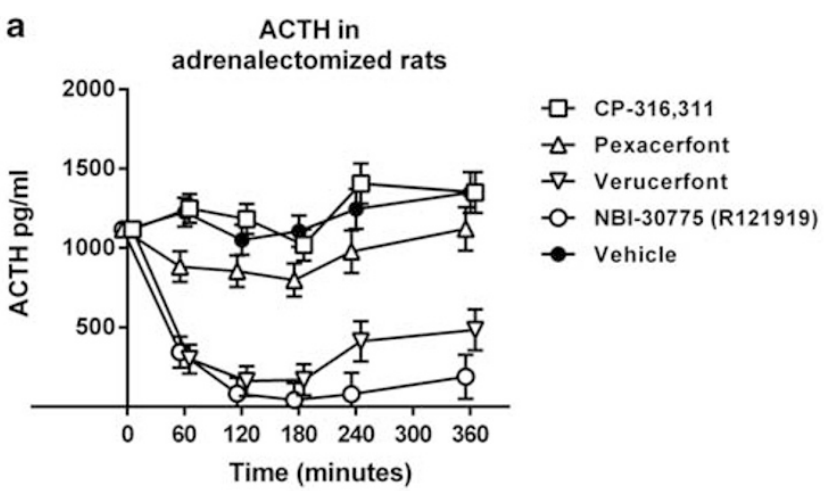

b

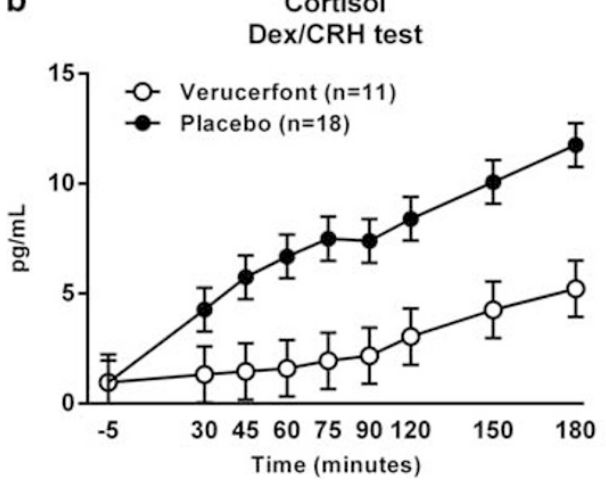

C

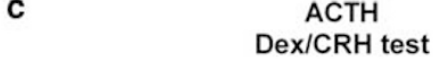

Figure 2 (a) Inhibition of plasma adrenocorticotropic hormone (ACTH) in adrenalectomized rats following a single dose of corticotropin-releasing factor receptor I (CRFI) antagonists with slow (NBI-30775, verucerfont) but not fast (CP-3 I 6,3 I I, pexacerfont) receptor dissociation rates. Adrenalectomized rats were administered vehicle or the respective antagonist immediately after a baseline sample $(t=0)$ was obtained, and plasma ACTH was sampled over the following $6 \mathrm{~h}$. Data points for the different drugs at each time-point have been jittered by \pm 5 min to allow visual separation, and represent covariate adjusted means \pm S.E.M., with baseline as covariate $(n=6-7)$. Non-adjusted baseline means were in the range $820-1215 \mathrm{pg} / \mathrm{ml}$. NBI-30775 and verucerfont suppressed $(P<0.0 \mathrm{I}) \mathrm{ACTH}$ at each time-point, both compared with the corresponding time-point for vehicle, and compared with their respective baseline. For detailed statistics, see Results. Blockade of (b) cortisol and (c) ACTH responses to dexamethasone/CRF challenge by verucerfont $(n=1 \mathrm{I})$ but not placebo $(n=18)$ in the clinical study. Data points represent mean \pm S.E.M. Covariates in the model included neuroticism and the total score from the Addiction Severity Index (ASI) for cortisol, and race, neuroticism, and total score from the ASI for ACTH. For detailed statistics, see Results.

this assay confirms that it can both bind and functionally inhibit the CRF1 receptor in vivo, and that this inhibition results in a robust and sustained inhibition of ACTH release upon stimulation by high CRF drive.

\section{Clinical Study}

Baseline characteristics of participants are summarized in Table 1. Randomization groups did not differ significantly on any of the characteristics listed.

Cortisol and dex-CRF test. There was no effect of verucerfont on morning cortisol values (data not shown). In response to the dex-CRF challenge, there were significant main effects of time on both cortisol $(\mathrm{F}[8,213]=14.77$, $P<0.0001$; Figure $2 \mathrm{~B})$ and ACTH levels $(\mathrm{F}[8,209]=3.53$, $P=0.0007$; Figure 2C), with levels increasing over time for both measures. In both cases, subjects receiving verucerfont showed a marked blunting of the HPA axis response to the dex-CRF challenge. There was a significant main effect of treatment on cortisol $(\mathrm{F}[1,28]=9.42 ; P=0.005)$ and a significant treatment by time interaction for $\mathrm{ACTH}$ $(\mathrm{F}[8,209]=2.17 ; P=0.03)$.

\section{Guided Imagery}

Craving responses. Exposure to guided imagery scripts reliably induced craving, as measured by the AUQ (Figure 3a). Specifically, there was a significant main effect of time $(F[7,226]=3.03, \quad P=0.005)$ such that craving increased significantly during the first $5 \mathrm{~min}$ of script presentation, and a significant time $\times$ script-type interaction $(F[14,407]=0.05, P=0.01)$. Post hoc analysis showed that craving at $5 \mathrm{~min}$ was higher compared with baseline ( $-15 \mathrm{~min})$ during both the alcohol script and the stress script, but not during the neutral script. There was, however, no main effect of verucerfont treatment on craving in response to the stress $(\mathrm{F}[1,34]=0.04, P=0.84$; Figure $3 \mathrm{~b})$ nor the alcohol script $(\mathrm{F}[1,36]=2.45, P=0.13$; Figure $3 \mathrm{c})$.

Anxiety responses. Script exposure also induced anxiety responses, as measured by the STAI (Figure 4a). Again, there was a significant main effect of time $(F[7,226]=2.14$, $P=0.04$ ), and a significant time $\times$ script-type interaction (F $[14,401]=4.77, P<0.0001)$. Post hoc analysis showed that anxiety ratings at $5 \mathrm{~min}$ were significantly elevated over baseline only during the stress script. There was, however, no 
Table I Subject Characteristics

\begin{tabular}{|c|c|c|c|c|}
\hline & $\begin{array}{l}\text { Verucerfont } \\
\quad(n=18)\end{array}$ & $\begin{array}{l}\text { Placebo } \\
(n=2 I)\end{array}$ & $\begin{array}{c}\text { Total } \\
\left(n=39^{a}\right)\end{array}$ & $P$-value ${ }^{b}$ \\
\hline \multicolumn{5}{|l|}{ Demographics } \\
\hline Age (years) & $40.1(12.9)$ & $45.5(10.8)$ & $43.0(12.0)$ & 0.16 \\
\hline Caucasian $^{c}$ & $13(76.5 \%)$ & $10(47.6 \%)$ & $23(60.5 \%)$ & 0.07 \\
\hline Education (years) & $13.8(2.4)$ & | $4.5(2.7)$ & $14.2(2.5)$ & 0.37 \\
\hline Family history density $^{d}$ & $0.3(0.3)$ & $0.3(0.2)$ & $0.3(0.2)$ & 0.69 \\
\hline \multicolumn{5}{|l|}{ Alcohol use (past 90 days) } \\
\hline Avg. drinks/drinking day & $12.7(5.3)$ & I I.2(6.4) & $11.9(5.9)$ & 0.43 \\
\hline Current anxiety disorder & $14(77.8 \%)$ & $16(76.2 \%)$ & $30(76.9 \%)$ & 0.91 \\
\hline Current mood disorder & $10(55.6 \%)$ & $10(47.6 \%)$ & $20(51.3 \%)$ & 0.62 \\
\hline Current DSM IV substance use disorder ${ }^{\mathrm{e}}$ & $5(27.8 \%)$ & $4(19.1 \%)$ & $9(23.1 \%)$ & 0.52 \\
\hline Current PTSD & $10(55.6 \%)$ & $7(33.3 \%)$ & $17(43.6 \%)$ & 0.16 \\
\hline CTQ total score & $62.1(28.6)$ & $48.2(20.1)$ & $54.6(25.1)$ & 0.10 \\
\hline Neuroticism score & $61.4(9.7)$ & $56.7(\mid 1.0)$ & $58.9(10.5)$ & 0.17 \\
\hline STAI-Trait score & $56.8(6.2)$ & $56.9(9.3)$ & $56.8(7.9)$ & 0.98 \\
\hline ASI total score & $2.4(0.6)$ & $2.1(0.6)$ & $2.2(0.6)$ & 0.25 \\
\hline
\end{tabular}

Abbreviations: ADS, Alcohol Dependence Scale; ASI, Addiction Severity Index; CTQ, Childhood Trauma Questionnaire; FTQ, Family Tree Questionnaire; PTSD, posttraumatic stress disorder, STAI, Spielberger State-Trait Anxiety Inventory; Trier/CR, Trier Social Stress/cue-reactivity test.

Variables are means (S.D.), or $n$ (percentage) as appropriate.

${ }^{a}$ Of the 39 total subjects, 4 completed the Trier/CR but did not complete the Scripts challenge.

${ }^{b}$ Based on a $\chi^{2}$ test for categorical measures, and a $t$-test for continuous measures.

'The remaining subjects were Black/African American.

${ }^{\mathrm{d}}$ Calculated as the proportion of first- and second-degree relatives known to have been treated for alcohol abuse or have alcohol use-related problems, as measured by the FTQ.

eSubstances other than alcohol.

significant effect of treatment on anxiety ratings in response to the stress script $(\mathrm{F}[1,36]=0.59, P=0.45$; Figure $4 \mathrm{~b})$ or the alcohol script $(\mathrm{F}[1,38]=0.1 .47, P=0.23$; Figure $4 \mathrm{c})$.

Neuroendocrine responses. In agreement with our prior findings, script exposure did not significantly activate the HPA axis (Kwako et al, 2015); (Supplementary Figures S1 and S2). Specifically, there was no main effect of script type $(\mathrm{F}[2,54]=0.24, P=0.79)$ or time $(\mathrm{F}[8,175]=1.49, P=0.16)$ on cortisol levels (Supplementary Figure S1A). Although there was a significant script type $\times$ time interaction $(\mathrm{F}[16$, $335]=1.72, P=0.03)$ in the mixed-effects model, none of the post hoc tests for cortisol levels were significant. Cortisol levels did not differ as a function of treatment during either the stress script $(\mathrm{F}[1,28]=1.13, P=0.30$; Supplementary Figure $\mathrm{S} 1 \mathrm{~B})$ or the alcohol script $(\mathrm{F}[1,30]=2.51, P=0.12$; Supplementary Figure S1C). There was a main effect of time $(\mathrm{F}[8,165]=3.11, P=0.003)$, but no main effect of script type $(F[2,49]=0.06, P=0.94)$ nor any script type $\times$ time interaction $(\mathrm{F}[16,338]=1.48, P=0.10)$ on the ACTH responses to the scripts (Supplementary Figure S2A). Similarly, ACTH levels did not differ as a function of treatment during either the stress script $(\mathrm{F}[1,29]=0.06, P=0.80$; Supplementary Figure S2B) or the alcohol script $(\mathrm{F}[1,27]=2.74, P=0.11$; Supplementary Figure S2C).

\section{Trier/Cue Reactivity}

Craving responses. There was a significant main effect of time $(F[4,209]=9.38, P<0.0001$; Figure 5a), such that craving for alcohol was significantly increased over baseline at $40 \mathrm{~min}$. There was no significant effect of verucerfont on craving in response to the Trier/CR $(\mathrm{F}[1,49]=1.30, P=0.26)$.

Anxiety responses. There was a significant main effect of time $(F[4,147]=14.23, P<0.0001$; Figure $5 b)$, such that anxiety ratings were significantly increased over baseline at $20 \mathrm{~min}$. There was a main effect of verucerfont on anxiety in response to the Trier/CR $(\mathrm{F}[1,46]=5.53 P=0.02)$; contrary to the hypothesis, however, subjects receiving verucerfont showed higher anxiety throughout the challenge compared with subjects receiving placebo. 

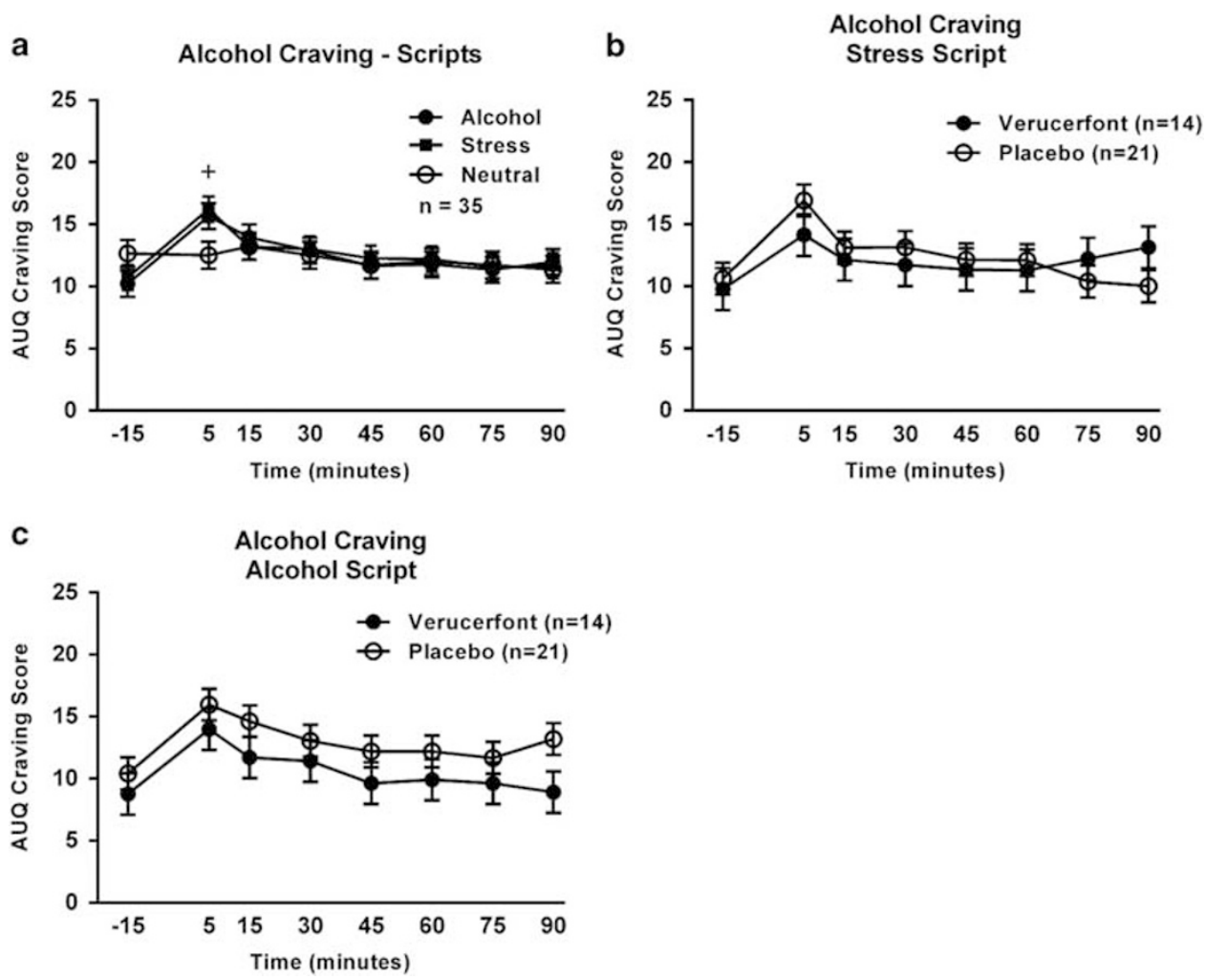

Figure 3 Alcohol craving responses to the guided imagery challenge session. (a) Effect of script type on alcohol craving. Data are mean \pm S.E.M.; $n=35$. Covariates in the model included age, neuroticism score from the NEO, and lifetime diagnosis of post-traumatic stress disorder (PTSD). The ' + ' indicates a significant difference between the 5 min and -15 min time points (Tukey's, $P<0.05$ ) for both the alcohol and stress scripts. (b) Effect of verucerfont on craving response to the stress script (verucerfont: $n=14$; placebo: $n=2 I$ ). Data points represent mean \pm S.E.M. Covariates in the model included race, neuroticism score, and total score from the Addiction Severity Index (ASI). (c) Effect of verucerfont on craving response to the alcohol cue script (verucerfont: $n=14$; placebo: $n=21$ ). Data points represent mean \pm S.E.M. Covariates in the model included race, average number of drinks per drinking day from the Timeline Follow-Back (TLFB), neuroticism score, and total score from the ASI. For detailed statistics, see Results.

Neuroendocrine responses. There was a significant main effect of time $(\mathrm{F}[8,260]=10.6, P<0.0001$; Figure 5C) on cortisol levels, such that cortisol was significantly increased over baseline by $40 \mathrm{~min}$. A similar effect was observed for ACTH (main effect of time: $\mathrm{F}[8,270]=16.5, P<0.0001$; Figure 5d), with ACTH levels significantly increased over baseline by $20 \mathrm{~min}$. There was no effect of verucerfont on either cortisol $(\mathrm{F}[1,42]=0.40, P=0.53)$ or ACTH levels $(\mathrm{F}[1,51]=0.01, P=0.98)$.

\section{fMRI}

As a primary candidate biomarker of its potential antistress action, we examined the ability of verucerfont to attenuate brain responses to negative emotional stimuli, because attenuation of amygdala responses to stress by CRF1 antagonists in rats effectively attenuates alcohol consumption. To validate this first fMRI biomarker, we analyzed responses to fearful faces in the placebo group. This analysis identified an expected activation within the amygdala, primarily on the right side (Figure 6, upper row), consistent with what has previously been reported for this type of stimuli (see, e.g., Hariri et al, 2002; Kwako et al, 2015). This response was blunted in the verucerfont group (Figure 6, middle row). A direct comparison between the groups showed a whole-brain significant attenuation of the response to fearful faces by verucerfont in a cluster located within the right amygdala $(P=0.03$ FWE-corrected, 91-voxel cluster size; see Figure 6, lower row, which provides the Talairach coordinates for the center of the cluster). Analyzing patterns of responses to negative IAPS did not reveal any clusters that withstood FWE-error correction. For the responses to alcohol-related stimuli, whole-brain analysis yielded variable results. A comparison of verucerfont and placebo treatment identified both clusters of attenuated responses, and those where the response was increased. The latter included some regions typically associated with alcohol and drug craving, such as the anterior cingulate (Supplementary Table T1).

\section{Tolerability}

Of the 44 subjects enrolled in the protocol, 9 were discontinued at some point during the study (4 of them completed the Trier/CR before being withdrawn, and thus were included in that analysis). Discontinuation rates were significantly higher in the verucerfont group $(8 / 22$ vs $1 / 22$, $X^{2}=6.8, P=0.009$ ). In the placebo group, one subject withdrew to resume blood pressure medication she had stopped taking to participate in the study. In the verucerfont group, four subjects withdrew voluntarily owing to medical or personal reasons. The four remaining subjects were withdrawn, one due to episodes of purging behavior, one due to a possible allergic reaction to study medication, one after testing positive for substance use while at the $\mathrm{NIH}$, and one 
a

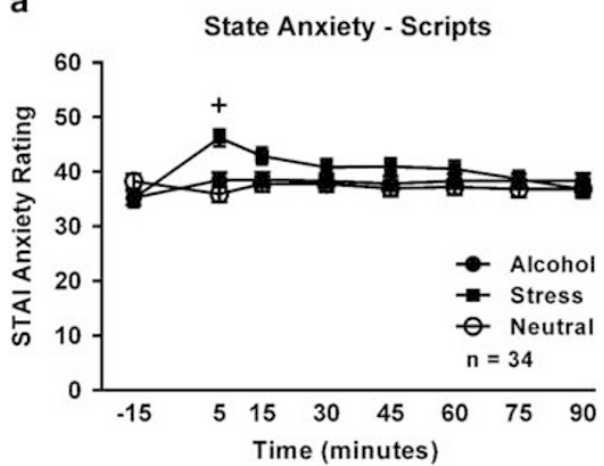

C

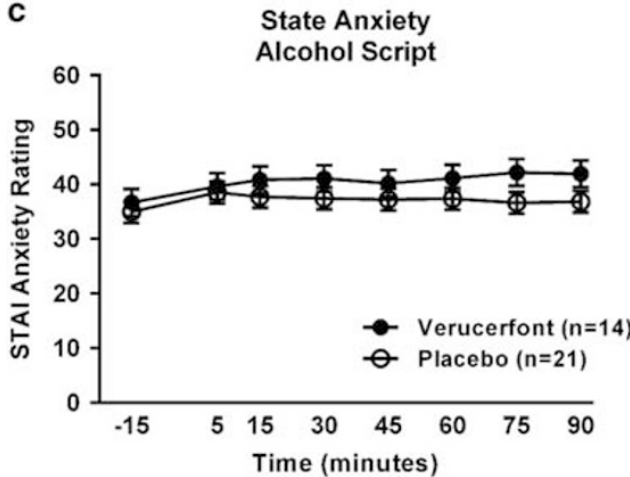

b

State Anxiety

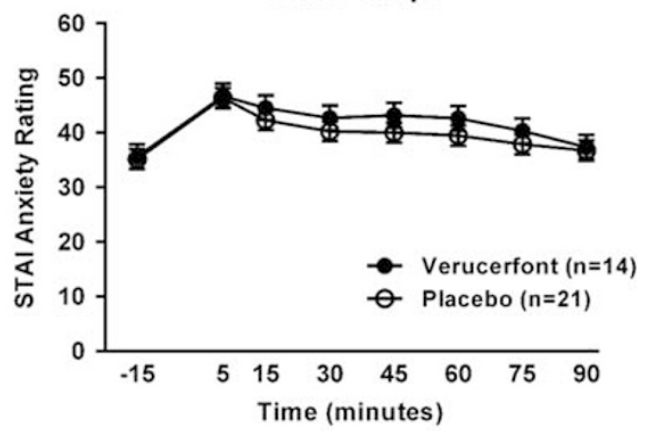

Figure 4 Anxiety response to the guided imagery challenge session. (a) Effect of script type on anxiety. Data points represent mean \pm S.E.M. Covariates in the model included age, race, years of education, neuroticism, total score on the Addiction Severity Index (ASI), and the trait anxiety score from the Spielberger State Trait Anxiety Inventory-Trait (STAI-Trait) version. One subject was missing the STAI-Trait version score, thus the sample size was reduced to 34 for this analysis. The ' + ' indicates a significant difference between the 5 min and -15 min time points (Tukey's, $P<0.05)$ during the stress script. (b) Effect of verucerfont on anxiety during the stress script (verucerfont: $n=14$; placebo: $n=21$ ). Data points represent mean \pm S.E.M. Covariates in the model included age, years of education, Alcohol Dependence Scale (ADS) score, and neuroticism. (c) Effect of verucerfont on anxiety during the alcohol cue script (verucerfont: $n=14$; placebo: $n=21$ ). Data points represent mean \pm S.E.M. Age was a covariate in the model. For detailed statistics, see Results.

due to a severe dissociative reaction in response to the stress script. The latter patient was a 47-year-old African-American female with comorbid PTSD.

\section{DISCUSSION}

We evaluated verucerfont for its ability to block HPA-axis activity in rats and humans, and for its ability to impact biomarkers that may be predictive of clinical efficacy in anxious $\mathrm{AD}$. In the clinical study, we used a battery that included neuroendocrine, behavioral, and brain imagingbased measures. Both the design and the outcome measures were similar to those recently used to evaluate another CRF1 antagonist, pexacerfont (Kwako et al, 2015). Verucerfont blocked HPA-axis activity in the adrenalectomized rat assay. Similarly, it blocked HPA-axis activation in the dex-CRF test in $\mathrm{AD}$ patients, preventing both ACTH and cortisol release that followed CRF stimulation. This was associated with attenuated brain responses to negative emotional stimuli. However, despite the antistress effects on HPA-axis and amygdala responses, the drug did not suppress experimental measures of alcohol craving or negative emotionality. Verucerfont was also less well tolerated than placebo, as evidenced by a significantly higher dropout rate.

A key finding of our study is to identify a translational biomarker of in vivo CRF1 antagonist activity. Although neither unstimulated HPA-axis output nor moderate-level output in response to a psychological challenge were affected by verucerfont, the antagonist potently blocked the HPA-axis response to an exogenous, supraphysiological CRF challenge. This effect was robust, and was reliably detected despite only a subset of participants undergoing the dex-CRF test because of a prolonged period of a nationwide pharmacy shortage in CRF. The in vivo HPA-axis suppressing activity of verucerfont in patients was predicted by rat data, which showed a potent and lasting suppression of ACTH output when a maximal activation of CRF drive was induced through adrenalectomy. Utility of the adrenalectomized rat assay for predicting in vivo CRF1 antagonist activity is further supported by the observations that another CRF1 antagonist, pexacerfont (Zhou et al, 2012), lacked activity in the rat assay, and was similarly unable to block the dex-CRF response in $\mathrm{AD}$ patients in a previous study (Kwako et al, 2015).

We cannot exclude the possibility that the difference in ability to block HPA-axis activation between verucerfont and pexacerfont could be related to sex differences; the pexacerfont study had predominantly male participants, whereas the verucerfont study was carried out exclusively in females. However, this appears unlikely to explain the findings, because the same difference between pexacerfont and verucerfont was observed in rats, using male subjects only. In fact, the difference in activity of pexacerfont and verucerfont in humans was predicted by a recently postulated 

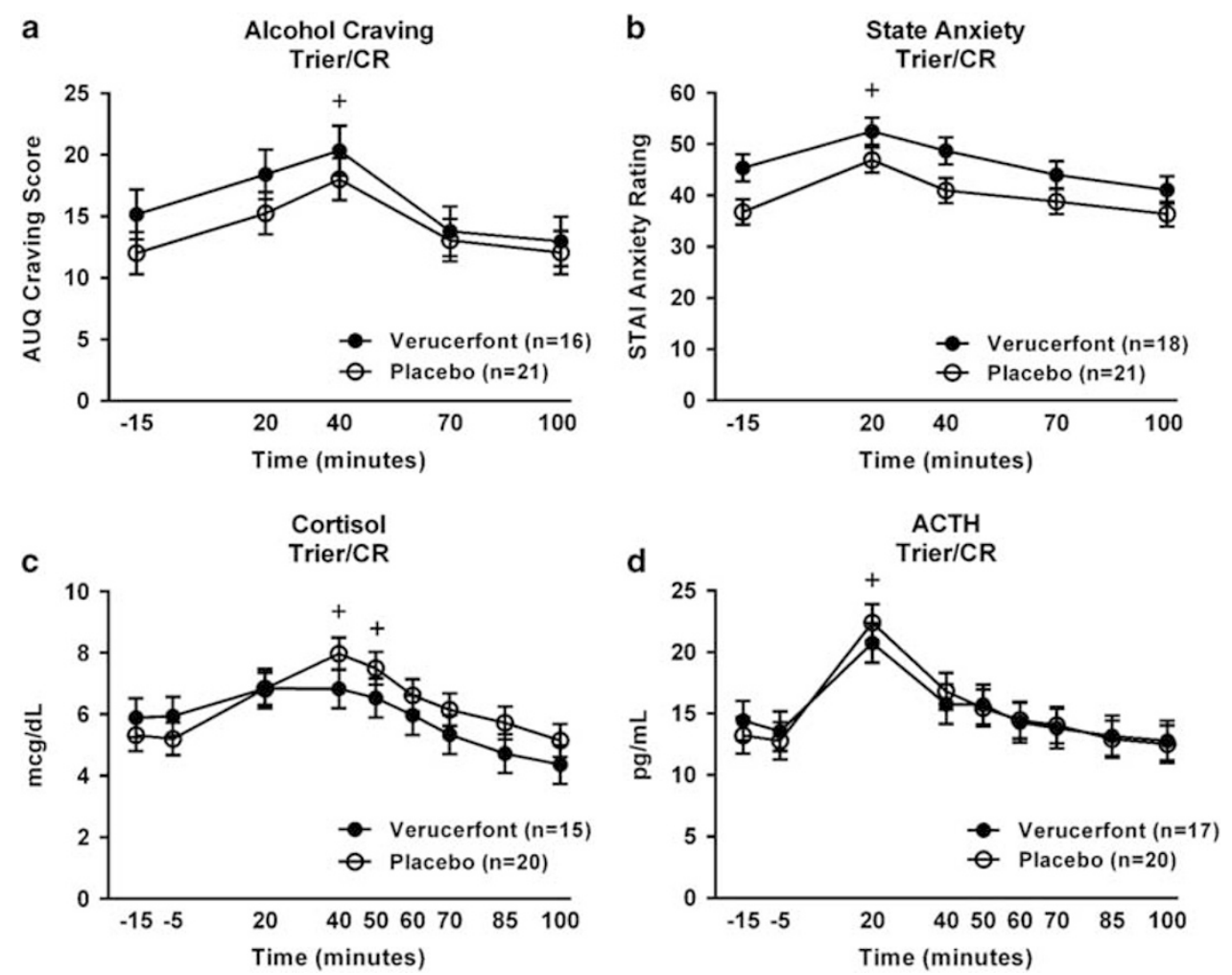

Figure 5 (a) Effect of verucerfont treatment on craving response to the Trier Social Stress/cue-reactivity test (Trier/CR) (verucerfont: $n=$ I6; placebo: $n=21$ ). Data points represent mean \pm S.E.M. Covariates in the model included race, average number of drinks per drinking day, and the total score from the Addiction Severity Index (ASI). The ' + ' indicates a significant difference between the 40 and -15 min time points (Tukey's $P<0.05)$. The sample sizes for the analyses of craving were reduced owing to missing data from the ASI for one subject, and missing data for race from another subject. (b) Effect of verucerfont treatment on anxiety during the Trier/CR (verucerfont: $n=18$; placebo: $n=21$ ). Data points represent mean \pm S.E.M. Covariates in the model included age and neuroticism. The ' + ' indicates an overall significant difference between the 20 and -15 min time points (Tukey's $P<0.05$ ); subjects receiving verucerfont showed higher anxiety overall compared with subjects receiving placebo. (c) Effect of verucerfont treatment on cortisol response to the Trier/CR (verucerfont: $n=15$; placebo: $n=20$ ). Data points represent mean \pm S.E.M. Covariates in the model included age, race, and total score from the ASI. The ' + ' indicates a significant difference from - 15 min time point at both 40 and 50 min (Tukey's $P<0.05$ ). (d) Effect of verucerfont treatment on adrenocorticotropic hormone $(A C T H)$ response to the Trier/CR (verucerfont: $n=17$; placebo: $n=20$ ). Data points represent mean \pm S.E.M. Covariates in the model included age and total score from the Childhood Trauma Questionnaire (CTQ). The '+' indicates a significant difference between the 20 min and - 15 min time points (Tukey's $P<0.05)$. For detailed statistics, see Results.

hypothesis (Fleck et al, 2012), according to which differences in dissociation rates from the CRF1 are a key property that determines in vivo activity of antagonists with similar nominal binding affinity to the receptor. In that context, pexacerfont has a fast off-rate from the receptor, predicting low functional activity, whereas verucerfont belongs to a family of molecules with slow off-rates that are predicted to be highly active (Fleck et al, 2012; Zorrilla et al, 2013).

Despite its antistress effects on HPA and amygdala responses, verucerfont did not influence behavioral measures of distress or anxiety. This observation is in agreement with a prior report (Grillon et al, 2015). Furthermore, and most importantly, verucerfont did not influence the primary outcome of the present study, stress-induced alcohol craving. Furthermore, we observed increased rather than suppressed brain responses to alcohol cues in some brain structures associated with alcohol craving, such as the anterior cingulate cortex. In summary, verucerfont thus did not produce the hypothesized signature of coordinated anticraving actions across a range of brain- and behavior-based biomarkers. This lack of activity is consistent with clinical results recently obtained with pexacerfont (Kwako et al, 2015), and stands in stark contrast to the animal literature with CRF1 antagonists in alcohol models (reviewed, e.g., in Heilig and Koob, 2007; Le and Shaham, 2002a; Zorrilla and Koob, 2013). Several potential explanations for these negative clinical findings should be considered.

First, it is possible that sufficient central CRF1 blockade was not achieved in our study. This possibility cannot be addressed directly, as no PET ligand is available that would allow central CRF1 occupancy by verucerfont to be determined. Nevertheless, this possibility appears unlikely, given that the dose regimen we used potently influenced the neuroendocrine biomarker. In fact, while not representing a direct in vivo proof of engagement, the results on ACTH and cortisol blockade after dex-CRF test may represent a proximal substrate-product biomarker, an alternative classic methodology for determining target engagement in humans (Simon et al, 2013). The relationship between pituitary and central receptor exposure to verucerfont in humans is not known, but animal experiments have indicated a favorable brain/plasma ratio of verucerfont (1.6×; Tellew et al, 2010), arguing against insufficient brain exposure when potent HPA-axis effects are observed. 


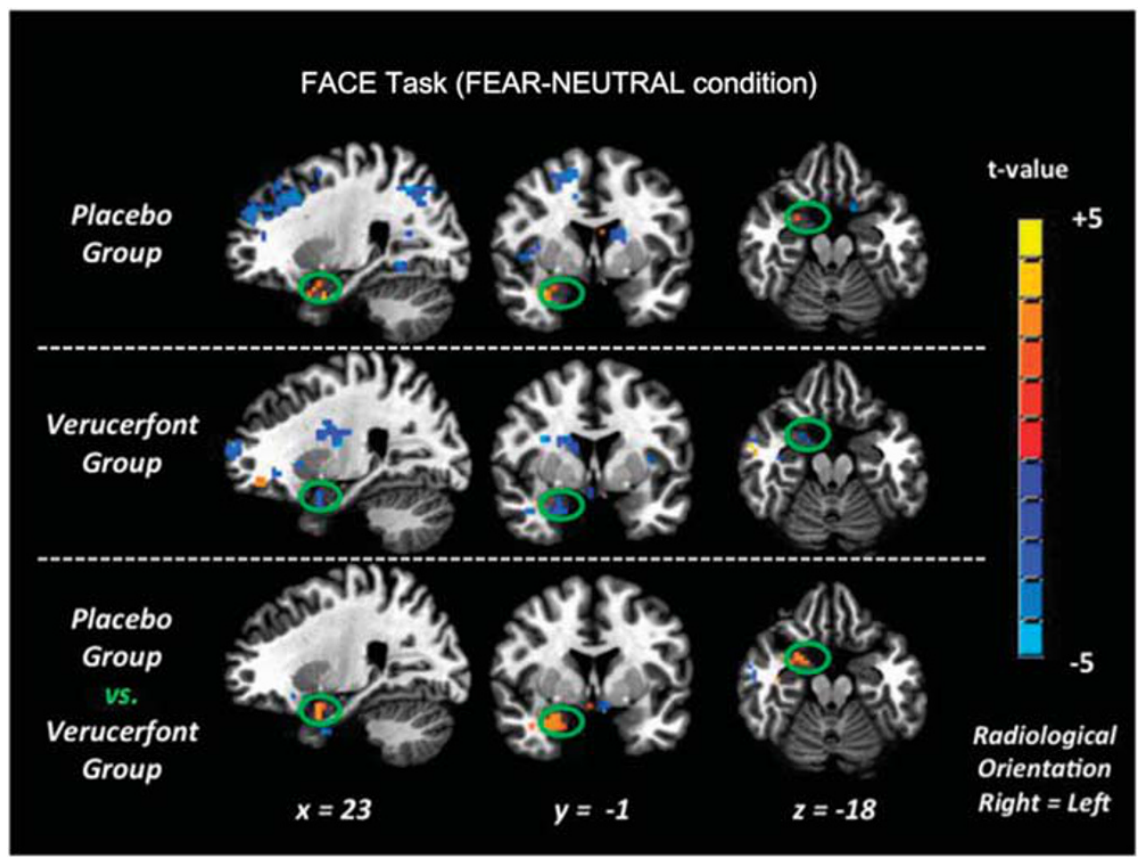

Figure 6 Functional magnetic resonance imaging blood oxygen level-dependent ( $\mathrm{MRR}$ BOLD) responses to fearful faces. Upper row: Right amygdala (within green oval) showed the predicted activation in the placebo group (placebo; $n=20$ ). Middle row: The corresponding activation in the verucerfont group was attenuated (verucerfont; $n=13$ ). Lower row: On direct comparison, there was a reduction in the verucerfont group, which exceeded the threshold for wholebrain corrected significance ( $P=0.03$ family-wise error (FWE)-corrected, 91 voxel cluster size). The whole-brain analysis model included the following covariates: race, ADS (Alcohol Dependence Scale); and CTQ (Childhood Trauma Questionnaire) scores.

Second, we cannot draw definitive conclusions regarding the clinical efficacy of verucerfont in alcoholism based on the current data, as our study measured experimental surrogate markers rather than actual stress-induced relapse to alcohol use. However, the possibility that the outcomes used are not suited to detect CRF1 antagonist activity also appears unlikely: although their properties and predictive power are not fully established, these measures have been shown to predict relapse (Sinha et al, 2011a), and to be sensitive to medications with activity in preclinical models of stress-induced relapse to alcohol seeking (Fox et al, 2012; George et al, 2008).

A third possibility to consider is that the study did not target the optimal patient population. In a heterogeneous condition such as $\mathrm{AD}$, characteristics of study participants may be decisive in determining whether an efficacy signal will be detected (Heilig et al, 2011; Litten et al, 2012; Sinha et al, 2011b). Whether craving and relapse are primarily driven by stress- or alcohol-associated stimuli, respectively, is a patient characteristic that has been suggested to predict treatment responses to different pharmacological mechanisms (Heinz et al, 2003). 'Reward craving' is sensitive to blockade by the approved alcohol medication naltrexone, whereas 'relief craving' is thought to be driven by the activity of systems that mediate behavioral stress responses (Le $e t$ al, 2002b; Liu and Weiss, 2002). 'Relief craving' has a prominent role in patients prone to anxiety and negative affect, a pattern commonly seen in female alcoholism where comorbidity with mood and anxiety disorders is particularly high (Grant et al, 2004; Kessler et al, 1997). Female patients with anxious $\mathrm{AD}$ should therefore be optimally sensitive to CRF1 blockers.

Finally, reports are accumulating in which studies have failed to detect activity of CRF1 antagonists in disease populations where animal work has predicted it, such as depression (Binneman et al, 2008; GlaxoSmithKline, 2010), anxiety disorders (Coric et al, 2010), or AD (Kwako et al, 2015; present study). If target engagement has, in fact, been achieved in these studies, appropriate patient populations have been targeted, and informative outcomes have been assessed, then the possibility must be considered that a consistent activity pattern of CRF1 antagonists in preclinical behavioral assays does not translate between rodents and humans. If that is the case, the reasons are presently unknown. We believe that the data we report here add an important piece of information to help establish whether this situation is, in fact, at hand.

\section{FUNDING AND DISCLOSURE}

The authors declare no conflict of interest.

\section{ACKNOWLEDGMENTS}

We want to thank NIAAA staff and NIH Clinical Center nursing staff for technical support. This study was supported by the NIAAA DICBR, and carried out under a Cooperative Research and Development Agreement (CRADA) between the NIAAA and GlaxoSmithKline.

\section{REFERENCES}

Asberg M, Schalling D (1979). Construction of a new psychiatric rating instrument, the Comprehensive Psychopathological Rating Scale (CPRS). Progr Neuro-Psychopharmacol 3: 405-412.

Bernstein DP, Fink L, Handelsman L, Foote J, Lovejoy M, Wenzel K et al (1994). Initial reliability and validity of a new retrospective 
measure of child abuse and neglect. Am J Psychiatry 151: 1132-1136.

Binneman B, Feltner D, Kolluri S, Shi Y, Qiu R, Stiger T (2008). A 6-week randomized, placebo-controlled trial of CP-316,311 (a selective CRH1 antagonist) in the treatment of major depression. Am J Psychiatry 165: 617-620.

Bohn MJ, Krahn DD, Staehler BA (1995). Development and initial validation of a measure of drinking urges in abstinent alcoholics. Alcohol Clin Exp Res 19: 600-606.

Brandon TH, Vidrine JI, Litvin EB (2007). Relapse and relapse prevention. Annu Rev Clin Psychol 3: 257-284.

Coric V, Feldman HH, Oren DA, Shekhar A, Pultz J, Dockens RC et al (2010). Multicenter, randomized, double-blind, active comparator and placebo-controlled trial of a corticotropinreleasing factor receptor-1 antagonist in generalized anxiety disorder. Depress Anxiety 27: 417-425.

Costa PT, McCree RR (2002). NEO Personality Inventory-Revised (NEO PI-R). APA: Washington, DC.

Cox RW (1996). AFNI: software for analysis and visualization of functional magnetic resonance neuroimages. Comput Biomed Res 29: $162-173$.

First MB, Spitzer RL, Gibbon M, Williams JBW (1996). Structured Clinical Interview for DSM-IV Axis I Disorders, Clinician Version (SCID-CV). American Psychiatric Press: Washington, DC.

Flannery BA, Volpicelli JR, Pettinati HM (1999). Psychometric properties of the Penn Alcohol Craving Scale. Alcohol Clin Exp Res 23: 1289-1295.

Fleck BA, Hoare SR, Pick RR, Bradbury MJ, Grigoriadis DE (2012). Binding kinetics redefine the antagonist pharmacology of the corticotropin-releasing factor type 1 receptor. J Pharmacol Exp Ther 341: 518-531.

Foa EB, Riggs DS, Dancu CV, Rothbaum BO (1993). Reliability and validity of a brief instrument for assessing posttraumatic-stressdisorder. J Trauma Stress 6: 459-473.

Fox HC, Anderson GM, Tuit K, Hansen J, Kimmerling A, Siedlarz KM et al (2012). Prazosin effects on stress- and cue-induced craving and stress response in alcohol-dependent individuals: preliminary findings. Alcohol Clin Exp Res 36: 351-360.

George DT, Gilman J, Hersh J, Thorsell A, Herion D, Geyer C et al (2008). Neurokinin 1 receptor antagonism as a possible therapy for alcoholism. Science 319: 1536-1539.

Gilligan PJ, Clarke T, He L, Lelas S, Li YW, Heman K et al (2009). Synthesis and structure-activity relationships of 8-(pyrid-3-yl) pyrazolo[1,5-a]-1,3,5-triazines: potent, orally bioavailable corticotropin releasing factor receptor-1 (CRF1) antagonists. J Med Chem 52: 3084-3092.

GlaxoSmithKline (2010). Results Summary for CRS106139. A sixweek, multicenter, randomized, double-blind, placebo-controlled, parallel-group study evaluating the efficacy, safety, and tolerability of GSK561679 compared to placebo in female subjects, diagnosed with major depressive disorder. Available at: http:// download.gsk-clinicalstudyregister.com/files/ee490a01-84334cba-a027-9c486b866a67. (date last accessed 16 May 2016).

Grant BF, Stinson FS, Dawson DA, Chou SP, Dufour MC, Compton W et al (2004). Prevalence and co-occurrence of substance use disorders and independent mood and anxiety disorders: results from the National Epidemiologic Survey on Alcohol and Related Conditions. Arch Gen Psychiatry 61: 807-816.

Grillon C, Hale E, Lieberman L, Davis A, Pine DS, Ernst M (2015). The CRH1 antagonist GSK561679 increases human fear but not anxiety as assessed by startle. Neuropsychopharmacology 40: 1064-1071.

Hariri AR, Tessitore A, Mattay VS, Fera F, Weinberger DR (2002). The amygdala response to emotional stimuli: a comparison of faces and scenes. Neuroimage 17: 317-323.

Heilig M, Egli M (2006). Pharmacological treatment of alcohol dependence: target symptoms and target mechanisms. Pharmacol Ther 111: 855-876.
Heilig M, Goldman D, Berrettini W, O'Brien CP (2011). Pharmacogenetic approaches to the treatment of alcohol addiction. Nat Rev Neurosci 12: 670-684.

Heilig M, Koob GF (2007). A key role for corticotropin-releasing factor in alcohol dependence. Trends Neurosci 30: 399-406.

Heinz A, Lober S, Georgi A, Wrase J, Hermann D, Rey ER et al (2003). Reward craving and withdrawal relief craving: assessment of different motivational pathways to alcohol intake. Alcohol Alcohol 38: 35-39.

Hunt WA, Barnett LW, Branch LG (1971). Relapse rates in addiction programs. J Clin Psychol 27: 455-456.

Kenward MG, Roger JH (1997). Small sample inference for fixed effects from restricted maximum likelihood. Biometrics 53: 983-997.

Kessler RC, Crum RM, Warner LA, Nelson CB, Schulenberg J, Anthony JC (1997). Lifetime co-occurrence of DSM-III-R alcohol abuse and dependence with other psychiatric disorders in the National Comorbidity Survey. Arch Gen Psychiatry 54: 313-321.

Kirschbaum C, Pirke KM, Hellhammer DH (1993). The 'Trier Social Stress Test' - a tool for investigating psychobiological stress responses in a laboratory setting. Neuropsychobiology 28: 76-81.

Kwako LE, Schwandt ML, Sells JR, Ramchandani VA, Hommer DW, George DT et al (2014). Methods for inducing alcohol craving in individuals with co-morbid alcohol dependence and posttraumatic stress disorder: behavioral and physiological outcomes. Addict Biol 20: 733-746.

Kwako LE, Spagnolo PA, Schwandt ML, Thorsell A, George DT, Momenan R et al (2015). The corticotropin releasing hormone-1 (CRH1) receptor antagonist pexacerfont in alcohol dependence: a randomized controlled experimental medicine study. Neuropsychopharmacology 40: 1053-1063.

Lang PJ, Bradley MM, Cuthbert BN (1999). International Affective Picture System (IAPS): Technical Manual and Affective Ratings. The Center for Research in Psychophysiology, University of Florida: Gainesville, FL.

Le A, Shaham Y (2002a). Neurobiology of relapse to alcohol in rats. Pharmacol Ther 94: 137-156.

Le AD, Harding S, Juzytsch W, Fletcher PJ, Shaham Y (2002b). The role of corticotropin-releasing factor in the median raphe nucleus in relapse to alcohol. J Neurosci 22: 7844-7849.

Littell RC, Milliken GA, Stroup WW, Wolfinger RD, Schabenberge O (2006). SAS for Mixed Models. Second Edition. SAS Institute Inc.: Cary, NC.

Litten RZ, Egli M, Heilig M, Cui C, Fertig JB, Ryan ML et al (2012). Medications development to treat alcohol dependence: a vision for the next decade. Addict Biol 17: 513-527.

Liu X, Weiss F (2002). Additive effect of stress and drug cues on reinstatement of ethanol seeking: exacerbation by history of dependence and role of concurrent activation of corticotropinreleasing factor and opioid mechanisms. J Neurosci 22: 7856-7861.

Mann RE, Sobell LC, Sobell MB, Pavan D (1985). Reliability of a family tree questionnaire for assessing family history of alcohol problems. Drug Alcohol Depend 15: 61-67.

Mantsch JR, Baker DA, Funk D, Le AD, Shaham Y (2016). Stressinduced reinstatement of drug seeking: 20 years of progress. Neuropsychopharmacology 41: 335-356.

Matsumoto D, Ekman P (1988). Japanese and Caucasian Facial Expressions of Emotion and Neutral Faces (JACFEE and $J A C N e u F)$ vol. 401 Human Interaction Laboratory, University of California: San Francisco, CA.

McLellan AT, Luborsky L, Woody GE, O'Brien CP (1980). An improved diagnostic evaluation instrument for substance abuse patients. The Addiction Severity Index. J Nerv Ment Dis 168: 26-33.

Modell S, Lauer CJ, Schreiber W, Huber J, Krieg JC, Holsboer F (1998). Hormonal response pattern in the combined DEX-CRH test is stable over time in subjects at high familial risk for affective disorders. Neuropsychopharmacology 18: 253-262. 
O'Malley SS, Krishnan-Sarin S, Farren C, Sinha R, Kreek MJ (2002). Naltrexone decreases craving and alcohol self-administration in alcohol-dependent subjects and activates the hypothalamo-pituitaryadrenocortical axis. Psychopharmacology (Berl) 160: 19-29.

Rivier JE, Kirby DA, Lahrichi SL, Corrigan A, Vale WW, Rivier CL (1999). Constrained corticotropin releasing factor antagonists (astressin analogues) with long duration of action in the rat. J Med Chem 42: 3175-3182.

Rydmark I, Wahlberg K, Ghatan PH, Modell S, Nygren A, Ingvar M et al (2006). Neuroendocrine, cognitive and structural imaging characteristics of women on longterm sickleave with job stressinduced depression. Biol Psychiatry 60: 867-873.

Simon GM, Niphakis MJ, Cravatt BF (2013). Determining target engagement in living systems. Nat Chem Biol 9: 200-205.

Sinha R, Fox HC, Hong KI, Hansen J, Tuit K, Kreek MJ (2011a). Effects of adrenal sensitivity, stress- and cue-induced craving, and anxiety on subsequent alcohol relapse and treatment outcomes. Arch Gen Psychiatry 68: 942-952.

Sinha R, Shaham Y, Heilig M (2011b). Translational and reverse translational research on the role of stress in drug craving and relapse. Psychopharmacology (Berl) 218: 69-82.

Skinner HA (1984). Assessing alcohol-use by patients in treatment. Res Adv Alcohol Drug 8: 183-207.

Sobell MB, Sobell LC, Klajner F, Pavan D, Basian E (1986). The reliability of a timeline method for assessing normal drinker college students' recent drinking history: utility for alcohol research. Addict Behav 11: 149-161.
Spielberger CD, Gorsuch RL, Lushene RE (1970). STAI Manual for the Stait-Trait Anxiety Inventory ('Self-Evaluation Questionnaire'). Consulting Psychologists Press: Palo Alto, CA. 24pp.

Stasiewicz PR, Gulliver SB, Bradizza CM, Rohsenow DJ, Torrisi R, Monti PM (1997). Exposure to negative emotional cues and alcohol cue reactivity with alcoholics: a preliminary investigation. Behav Res Ther 35: 1143-1149.

Tellew JE, Lanier M, Moorjani M, Lin E, Luo Z, Slee DH et al (2010). Discovery of NBI-77860/GSK561679, a potent corticotropin-releasing factor (CRF1) receptor antagonist with improved pharmacokinetic properties. Bioorg Med Chem Lett 20: 7259-7264.

Wolpe J (1969). The Practice of Behavior Therapy, 1st edn. Pergamon Press: New York, NY, USA, 314pp.

Zhou L, Dockens RC, Liu-Kreyche P, Grossman SJ, Iyer RA (2012). In vitro and in vivo metabolism and pharmacokinetics of BMS-562086, a potent and orally bioavailable corticotropinreleasing factor-1 receptor antagonist. Drug Metab Dispos 40: 1093-1103.

Zobel AW, Nickel T, Kunzel HE, Ackl N, Sonntag A, Ising M et al (2000). Effects of the high-affinity corticotropin-releasing hormone receptor 1 antagonist R121919 in major depression: the first 20 patients treated. J Psychiatr Res 34: 171-181.

Zorrilla EP, Heilig M, de Wit H, Shaham Y (2013). Behavioral, biological, and chemical perspectives on targeting $\operatorname{CRF}(1)$ receptor antagonists to treat alcoholism. Drug Alcohol Depend 128: $175-186$.

Supplementary Information accompanies the paper on the Neuropsychopharmacology website (http://www.nature.com/npp) 